\title{
An Efficient Object Tracking Algorithm in Wireless Sensor Networks
}

\author{
Cheng-Ta Lee ${ }^{1,2}$, Frank Yeong-Sung Lin ${ }^{1}$, and Yean-Fu Wen ${ }^{1,3}$ \\ Department of Information Management \\ ${ }^{1}$ National Taiwan University \\ ${ }^{2}$ Lan Yang Institute of Technology \\ ${ }^{3}$ China University of Technology \\ Taipei, Taiwan, R.O.C. \\ \{d90001, yslin, d89002\}@im.ntu.edu.tw
}

\begin{abstract}
In this paper, we develop a heuristic algorithm to construct an efficient object tracking in wireless sensor networks (WSNs). Such wireless sensor network has to be designed to achieve efficient object tracking for given arbitrary topology of wireless sensor networks. We consider the two-way object moving frequency of each pair of sensor nodes and link transmission cost. This problem is formulated as $0 / 1$ integerprogramming problem. A Lagrangean Relaxationbased (LR-based) heuristic algorithm is proposed for solving the optimization problem. The experimental results showed that the proposed algorithm gets a near optimization in the efficient object tracking. Furthermore, the algorithm is very efficient and scalable in terms of the solution time.
\end{abstract}

Keywords: Wireless sensor networks (WSNs), object tracking, Lagrangean Relaxation (LR).

\section{Introduction}

The rapid growth in sensor technology and wireless communication has resulted in the development of wireless sensor networks (WSNs). WSNs have benefits of low cost and wireless communication capability. It consists of several sensors, sink nodes, and back-end system. These sensors work in coordination with collect physical information from the sensor field, and they can process and forward the information to the sink nodes. Eventually, the back-ends can obtain global views according to the information provided by the sink nodes $[3,4]$.

Object tracking is key application issue of WSNs which are widely deployed for military intrusion detection and wildlife animal monitoring. Object tracking wireless sensor networks have two critical operations [7-9]. First is monitoring. Sensor nodes are required to detect and track the movement states of mobile object. Second is reporting. The nodes sensing the object need to report their discoveries to the sink. These two operations are interleaved during the entire object tracking process. Our focus, in previous studies $[1,2]$, have been on developing strategies for reducing the energy consumption in reporting operations.

In this paper, the prior studies are expanded to the efficient object tracking in wireless sensor networks. We focus on the problem of constructing an efficient wireless sensor networks for object tracking services using the object tracking tree. Therefore, we motivate to propose a heuristic algorithm to cope with the problem with a given wireless sensor network arbitrary topology, two-way object moving frequency of each pair of sensor nodes, and link transmission cost. The total communication cost can be computed and minimized by object tracking tree.

The calculating communication cost is different from $[1,2]$. First, we consider the two-way object moving frequency of each pair of sensor nodes because the round-trip object moving frequency of each pair of sensor nodes is different. Second we consider the link transmission cost. Figure 1 illustrates an example of calculating communication cost. The weight of each solid link represents link transmission cost between a pair of adjacent communication nodes or between a pair of sensor node and communication node, and the weight of each dash link represents the frequency of object movement between a pair of adjacent sensors. Communication cost is $70(10 * 7)$ when object moves from sensor $x$ to sensor $y$, and communication cost is $56((8+6) * 4)$ when object moves from sensor $y$ to sensor $x$. 


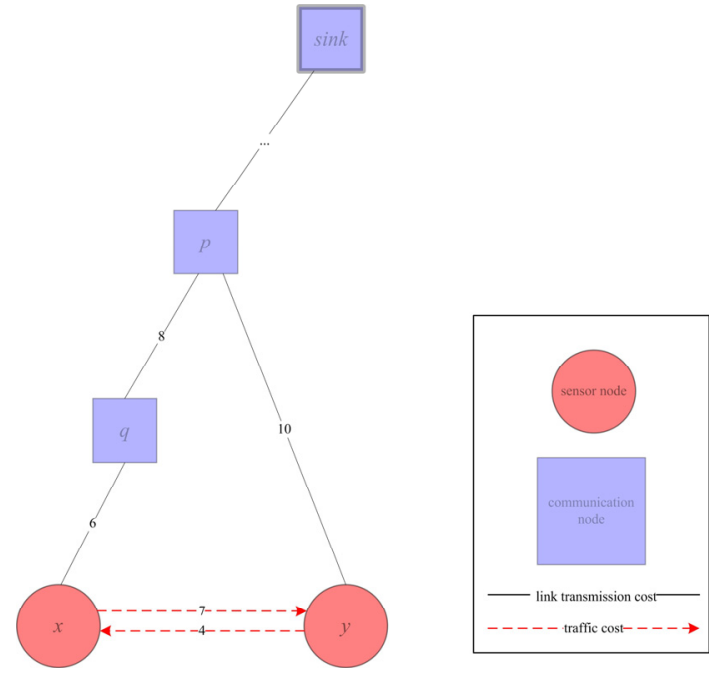

Fig. 1: Example of calculating communication cost.

In this paper, we formulate the problem as a $0 / 1$ integer programming problem where the objective function is the minimization of the total communication cost subject to routing, tree, and variable-transformation constraints. We use the Lagrangean Relaxation-based (LR-based) heuristic algorithm to solve the sub-problem and obtain a primal feasible solution.

The problem is formulated as a linear optimization-based problem with three different decision variables: paths, tree links, and tracking links. Paths are original-destination pair between sensor nodes and sink node. Tree links are the links of object tracking tree. Tracking links are the links when object moves from sensor $x$ to sensor $y$, and then sensor $y$ delivers tracking information upward to the first common ancestor via the tracking links [1]. For example, tracking links are the links between communication node $p$ and sensor node $y$ in Figure 1 . To fulfill the timing and the quality requirement of the optimal decisions, the Lagrangean Relaxation method, which has been successfully adopted to solve many famous NP-complete problems $[5,6]$, is used. In the further computational experiments, our proposed object tracking algorithm is expected to be efficient and effective in dealing with the complicated optimization problem.

From papers review, this study differs from the prior works in two points $[1,2]$. First, we consider the two-way object moving frequency of each pair of sensor nodes and link transmission cost. Second, we present a LR mathematical model to describe the optimization problem and LR-based heuristic algorithm is proposed to solve the problem.
The rest of this paper is organized as follows. The problem and mathematic model are described in section 2 and 3, respectively. Additionally, the solution procedure is presented in section 4 . Furthermore, the computational results are discussed in section 5, and conclusions are presented in section 6.

\section{Problem Description}

Our approach uses hierarchical object tracking tree to record information about presence of the object and to keep this information up to date. Sensor nodes are required to detect and track the movement states of mobile object. The information about presence of the detected object is stored at communication nodes, and each communication node particularly stores the set of object that was detected jointly by its descendants. This set is called the detected set. For example, the detected set of a sensor at a leaf node consists of just the objects within the sensor's detection range, while the root node's detected set contains all objects presented in the sensor field [1].

The efficient object tracking in WSNs problem is modeled as a graph, $G(V, L)$, where $V$ are sensor nodes and communication nodes (including sink node) distributed in a two-dimensional plane and link $L$ (e.g., $(i, j)$ represents that node $j$ is covered by $i$ 's radio), as shown in Figure 2 and 3.

For example, the sensor sub-graph in Figure 2 illustrates a 2D sensor field with each edge connecting a pair of adjacent sensors. Each link weight is object moving frequency of each pair of sensor nodes.

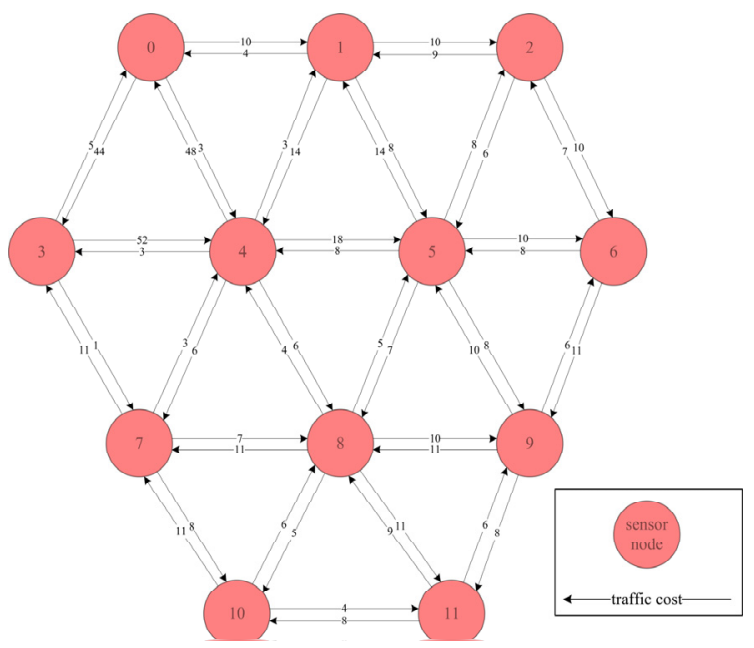

Fig. 2: Example of 2D sensor sub-graph. 
Figure 3 illustrates a 2D sensor field's tracking sub-graph with each edge connecting a pair of adjacent communication nodes or sensor-communication nodes. Each link weight represents link transmission cost.

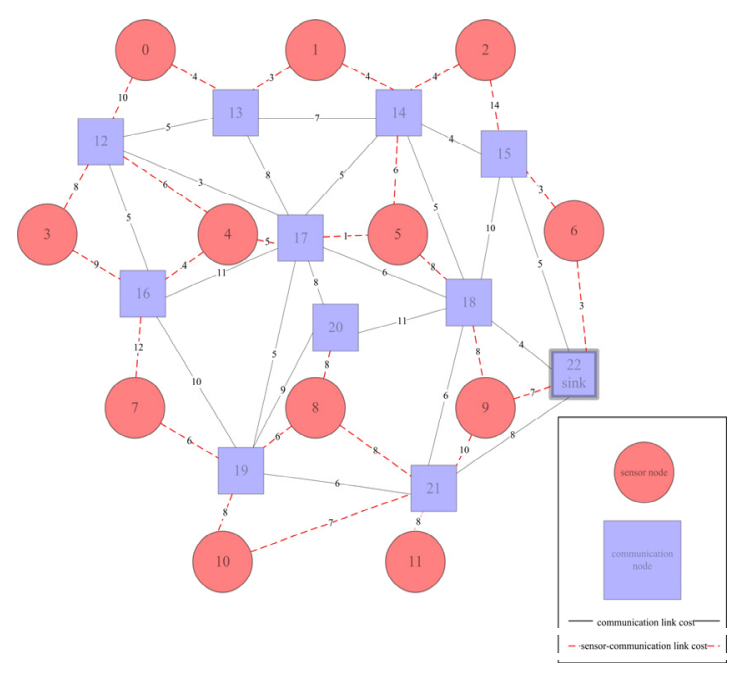

Fig. 3: Example of 2D tracking sub-graph.

Figure 4 illustrates a 2D sensor field's object tracking tree with each edge connecting a pair of adjacent nodes. Each link weight represents link transmission cost between a pair of adjacent communication nodes or sensor-communication nodes. The root is sink node. This special example is also a shortest path tree (SPT).

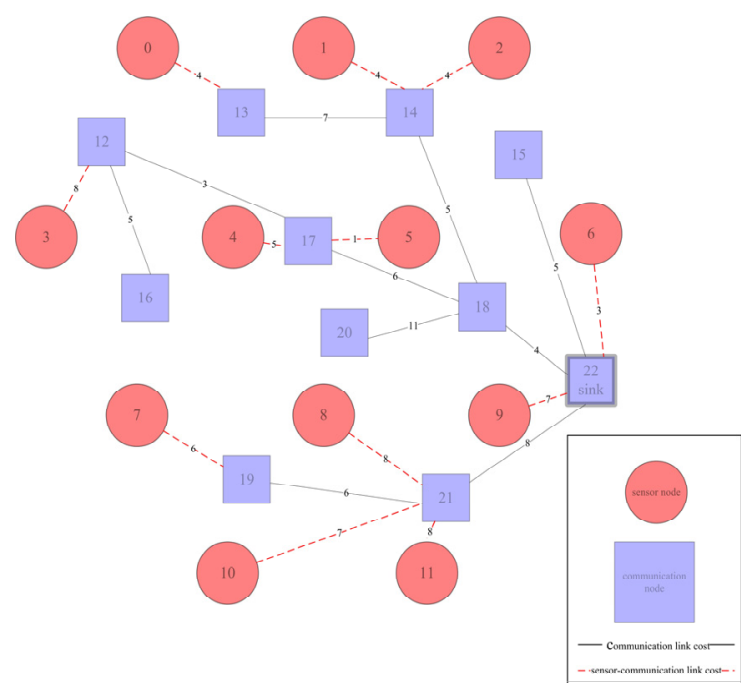

Fig. 4: Example of 2D object tracking tree.

In this paper, we consider a given wireless sensor network arbitrary topology, two-way object moving frequency of each pair of sensor nodes, and link transmission cost. The sensor field consists of sensor nodes and communication nodes. We deploy hierarchical network topology architecture. All sensor nodes send data to upper layer communication nodes. Eventually, the sensing information is sent to sink node.

A good tracking method is characterized by a low total communication cost [1]. Given a sensor graph and an object tracking tree, we can compute the total communication cost.

We define the total communication cost for a graph $G$ and object tracking tree $T$ as the sum of the individual donations of all pairs of adjacent sensor nodes in $G$ :

Total Communication Cost $(G, T)$

$$
=\sum \sum_{x \in S} \sum_{y \in S} t_{(i, j) \in L}^{x y} r_{x y} a_{(i, j)}
$$

Where $S$ is the set of all sensor nodes and $L$ is the set of all links. The decision variable $t_{(i, j)}^{x y}=1$ if $z_{(i, j)}^{x}=0 \bigcap z_{(i, j)}^{y}=1$ (reporting object's location uses the link $(i, j)$ when object moves from sensor $x$ to sensor $y$ ) and 0 otherwise, where $z_{(i, j)}^{s}=1$ if the sensor node $s$ uses the link $(i, j)$ to reach the sink node and 0 otherwise. $r_{x y}$ is the frequency of object movement from $x$ to $y$ and $a_{(i, j)}$ is the transmission cost associated with link $(i, j)$.

\section{Problem Formulation}

The notations used to model the problem are listed as follows.

\begin{tabular}{|c|l|}
\hline \multicolumn{2}{|c|}{ Given Parameters } \\
\hline Notation & \multicolumn{1}{c|}{ Description } \\
\hline$S$ & The set of all sensor nodes. \\
\hline$C$ & $\begin{array}{l}\text { The set of all communication nodes, } \\
\text { including sink node. }\end{array}$ \\
\hline$R$ & $\begin{array}{l}\text { The set of the frequency of object } \\
\text { movement from } x \text { to } y, \forall x, y \in S, \\
x \neq y .\end{array}$ \\
\hline$L$ & The set of all links, $(i, j) \in L, i \neq j$. \\
\hline$A$ & $\begin{array}{l}\text { The set of transmission cost } a_{(i, j)} \\
\text { associated with link }(i, j) .\end{array}$ \\
\hline$P_{s}$ & $\begin{array}{l}\text { The set of all candidate paths } p \\
\text { between any pair }(s, \text { sink }), \forall s \in S .\end{array}$ \\
\hline \multicolumn{1}{|c|}{ Indicate Parameter } \\
\hline Notation & $\begin{array}{l}\text { The indicator function which is } 1 \text { if } \\
\text { link }(i, j) \text { is on path } p \text { and } 0 \\
\text { otherwise. }\end{array}$ \\
\hline$\delta_{p(i, j)}$
\end{tabular}




\begin{tabular}{|c|l|}
\hline \multicolumn{2}{|c|}{ Decision Variables } \\
\hline Notation & \multicolumn{1}{|c|}{ Description } \\
\hline \multirow{3}{*}{$x_{p}$} & $\begin{array}{l}1 \text { if the sensor node } s \text { uses the path } p \\
\text { to reach the sink node and 0 otherwise, } \\
\forall s \in S, p \in P .\end{array}$ \\
\hline \multirow{3}{*}{$z_{(i, j)}^{s}$} & $\begin{array}{l}1 \text { if the sensor node } s \text { uses the link } \\
(i, j) \text { to reach the sink node and 0 } \\
\text { otherwise. }\end{array}$ \\
\hline$t_{(i, j)}^{x y}$ & $\begin{array}{l}1 \text { if } z_{(i, j)}^{x}=0 \cap z_{(i, j)}^{y}=1 \text { (reporting } \\
\text { object's location uses the link }(i, j) \\
\text { when object moves from sensor } x \text { to } \\
\text { sensor } y \text { ) and 0 otherwise, } x \neq y .\end{array}$ \\
\hline
\end{tabular}

\section{Problem (IP):}

Objective function:

$$
Z_{I P}=\min \sum_{x \in S} \sum_{y \in S} \sum_{(i, j) \in L} t_{(i, j)}^{x y} r_{x y} a_{(i, j)}
$$

subject to:

$$
\begin{array}{ll}
\sum_{p \in P_{p}}^{x_{p}} x_{p} & \forall s \in S \\
\sum_{j \in C}^{s} z_{(i, j)}^{s}=1 & \forall s \in S, i \in S \cup C \\
\sum_{p \in P_{s}}^{x} x_{p} \delta_{p(i, j)} \leq z_{(i, j)}^{s} & -\{\operatorname{sink}\} \\
2 t_{(i, j)}^{x y} \leq z_{(i, j)}^{y}-z_{(i, j)}^{x}+1 & \forall x \in S,(i, j) \in L \in S,(i, j) \in L \\
z_{(i, j)}^{y}-z_{(i, j)}^{x}+1 \leq t_{(i, j)}^{x y}+1 & \forall x, y \in S,(i, j) \in L \\
\sum_{(i, j) \in L} t_{(i, j)}^{x y} \geq 1 & \forall x, y \in S \\
x_{p}=0 \text { or } 1 & \forall s \in S, \quad p \in P_{s} \\
z_{(i, j)}^{s}=0 \text { or } 1 & \forall s \in S,(i, j) \in L \\
t_{(i, j)}^{x y}=0 \text { or } 1 & \forall x, y \in S,(i, j) \in L .
\end{array}
$$

The objective function (IP) of this problem is to minimize the total communication cost subject to:

Constraint (1): Routing constraint which uses one path from sensor node $s$ to sink node only.

Constraint (2): Tree constraint of avoiding cycle. Any nodes' outgoing link to communication node is equal to 1 on the object tracking tree, except the sink node.

Constraint (3): Routing constraint. Once the path, $x_{p}$, is selected and the link $(i, j)$ is on the path, then the decision variable, $z_{(i, j)}^{s}$, must be enforced to equal 1.
Constraint (4-5): There are variable-transformation constraints. If $z_{(i, j)}^{x}=0 \bigcap z_{(i, j)}^{y}=1$, reporting object's location uses the link $(i, j)$ when object moves from sensor $x$ to sensor $\mathrm{y}, t_{(i, j)}^{x y}$ must be enforced to equal 1 and 0 otherwise.

Constraint (6): For all $\sum_{(i, j) \in L} t_{(i, j)}^{x y}$ must be greater than or equal to 1 . This is redundant constraint.

Constraint (7-9): Decision variables $x_{p}, \quad z_{(i, j)}^{s}$, and $t_{(i, j)}^{x y}$ equal 0 or 1.

\section{Solution Approach}

\subsection{Lagrangean Relaxation}

Using the Lagrangean Relaxation method, which has been successfully adopted to solve many famous NP-complete problems [5, 6], we can transform the primal problem (IP) into the following Lagrangean Relaxation problem (LR) where constraints (3), (4), and (5) are relaxed. For a vector of non-negative Lagrangean multipliers, a Lagrangean Relaxation problem of (IP) is given by

\section{Problem(LR):}

Objective function:

$Z_{L R}\left(u_{s(i, j)}^{1}, u_{x y(i, j)}^{2}, u_{x y(i, j)}^{3}\right)$

$=\min \left\{\sum_{x \in S} \sum_{y \in S} \sum_{(i, j) \in L} t_{(i, j)}^{x y} r_{x y} a_{(i, j)}\right.$

$+\sum_{s \in S} \sum_{(i, j) \in L} \sum_{p \in P_{s}} u_{s(i, j)}^{1} x_{p} \delta_{p(i, j)}$

$-\sum_{s \in S} \sum_{(i, j) \in L} u_{s(i, j)}^{1} z_{(i, j)}^{s}$

$+\sum_{x \in S} \sum_{y \in S} \sum_{(i, j) \in L} u_{x y(i, j)}^{2} 2 t_{(i, j)}^{x y}$

$-\sum_{x \in S} \sum_{y \in S} \sum_{(i, j) \in L} u_{x y(i, j)}^{2}\left(z_{(i, j)}^{y}-z_{(i, j)}^{x}+1\right)$

$+\sum_{x \in S} \sum_{y \in S} \sum_{(i, j) \in L} u_{x y(i, j)}^{3}\left(z_{(i, j)}^{y}-z_{(i, j)}^{x}+1\right)$

$\left.-\sum_{x \in S} \sum_{y \in S} \sum_{(i, j) \in L} u_{x y(i, j)}^{3}\left(t_{(i, j)}^{x y}+1\right)\right\}$

subject to: (1), (2), (6), (7), (8), and (9)

Where $u_{s(i, j)}^{1}, u_{x y(i, j)}^{2}$, and $u_{x y(i, j)}^{3}$ are Lagrangean multipliers and $u_{s(i, j)}^{1}, u_{x y(i, j)}^{2}$, and $u_{x y(i, j)}^{3} \geq 0$. To solve (LR), we can decompose (LR) into the following four independent and easily solvable optimization sub-problems.

$$
Z_{L R}=Z_{s u b 1}+Z_{s u b 2}+Z_{s u b 3}+Z_{s u b 4}
$$


Sub-problem 1: (related to decision variable $t_{(i, j)}^{x y}$ ) Objective function:

$$
\begin{aligned}
& Z_{s u b 1}\left(u_{x y(i, j)}^{2}, u_{x y(i, j)}^{3}\right) \\
& =\min \sum_{x \in S} \sum_{y \in S} \sum_{(i, j) \in L} t_{(i, j)}^{x y}\left(r_{x y} a_{(i, j)}+2 u_{x y(i, j)}^{2}-u_{x y(i, j)}^{3}\right)
\end{aligned}
$$

subject to: (6) and (9)

This sub-problem is related to decision variable $t_{(i, j)}^{x y}$, which can be further decomposed into $|S|^{2}|L|$ sub-problems.

Constraint (6) is redundant constraint used to reduce the duality gap.

Two cases are listed to determine the value of $t_{(i, j)}^{x y}$.

Let $\theta_{x y(i, j)}$ denote the weight of the a pair of sensor nodes $x$ and $y$ using the link $(i, j)$, we get

$$
\theta_{x y(i, j)}=\left(r_{x y} a_{(i, j)}+2 u_{x y(i, j)}^{2}-u_{x y(i, j)}^{3}\right)
$$

Case 1: If $\theta_{x y(i, j)}<0$, then assign $t_{(i, j)}^{x y}=1$

Case 2: If $\theta_{x y(i, j)} \geq 0$, then assign $t_{(i, j)}^{x y}=0$.

If the sum of each pair node $t_{(i, j)}^{x y}$ is zero, we enforce to select the minimum positive objective value $\theta_{x y(i, j)}$ and set $t_{(i, j)}^{x y}=1$ to fulfill the constraint (6).

Sub-problem 2: (related to decision variable $x_{p}$ ) Objective function:

$$
Z_{s u b 2}\left(u_{s(i, j)}^{1}\right)=\min \sum_{s \in S} \sum_{(i, j) \in L}\left(u_{s(i, j)}^{1} \sum_{p \in P} x_{p} \delta_{p(i, j)}\right)
$$

subject to: (1) and (7)

Sub-problem 2 is a shortest path problem. The value of $x_{p}$ can be determined by the link cost, $u_{s(i, j)}^{1}$. This sub-problem is related to decision variable $x_{p}$, which can be further decomposed into $|S||L|$ sub-problems.

Sub-problem 3: (related to decision variable $z_{(i, j)}^{s}$ ) Objective function:

$$
\begin{aligned}
& Z_{s u b 3}\left(u_{s(i, j)}^{1}, u_{x y(i, j)}^{2}, u_{x y(i, j)}^{3}\right) \\
& =\min \sum_{s \in S} \sum_{(i, j) \in L}\left[-u_{s(i, j)}^{1}+\sum_{x \in S}\left(u_{x s(i, j)}^{3}-u_{x s(i, j)}^{2}\right)\right. \\
& \left.-\sum_{y \in S}\left(u_{s y(i, j)}^{3}-u_{s y(i, j)}^{2}\right)\right] z_{(i, j)}^{s}
\end{aligned}
$$

\section{subject to: (2) and (8)}

This sub-problem is related to decision variable $z_{(i, j)}^{s}$, which can be further decomposed into $|S||L|$ sub-problems.
Let $\psi_{s(i, j)}$ denote the weight of the sensor node s using the link $(i, j)$, we get $\psi_{s(i, j)}=$

$$
-u_{s(i, j)}^{1}+\sum_{x \in S}\left(u_{x s(i, j)}^{3}-u_{x s(i, j)}^{2}\right)-\sum_{y \in S}\left(u_{s y(i, j)}^{3}-u_{s y(i, j)}^{2}\right)
$$

$z_{(i, j)}^{s}$ must be enforced to equal 1 when choosing the minimum of $\psi_{s(i, j)}$ for each $\mathrm{s}$ and $\mathrm{i}$ to fulfill the constraint (2).

\section{Sub-problem 4: (Constant Part)}

Objective function:

$$
Z_{s u b 4}\left(u_{x y(i, j)}^{2}\right)=-\sum_{x \in S} \sum_{y \in S} \sum_{(i, j) \in L} u_{x y(i, j)}^{2}
$$

According to the weak Lagrangean duality theorem [5, 6], $Z_{D}\left(u_{s(i, j)}^{1}, u_{x y(i, j)}^{2}, u_{x y(i, j)}^{3}\right)$ is a lower bound (LB) on $Z_{I P}$ when $u_{s(i, j)}^{1}, u_{x y(i, j)}^{2}$, and $u_{x y(i, j)}^{3} \geq 0$. The following dual problem (D) is then constructed to calculate the tightest lower bound.

\section{Dual Problem (D):}

Objective function:

$$
Z_{D}=\max Z_{D}\left(u_{s(i, j)}^{1}, u_{x y(i, j)}^{2}, u_{x y(i, j)}^{3}\right)
$$

\section{subject to:}

$$
u_{s(i, j)}^{1}, u_{x y(i, j)}^{2} \text {, and } u_{x y(i, j)}^{3} \geq 0
$$

There are several methods for solving the dual-mode problem (D). One of the most popular is the subgradient method.

\subsection{Getting Primal Feasible Solutions}

After optimally solving the Lagrangean dual problem, we get a set of decision variables and develop LR-based heuristic algorithm to tune these decision variables. A set of feasible solutions of the primal problem (IP) then can be obtained. The primal feasible solution is an upper bound (UB) of the problem (IP), and the Lagrangean dual problem solution guarantees the lower bound $(L B)$ of the problem (IP). Iteratively, by solving getting primal feasible solution and Lagrangean dual problem, we get the $U B$ and $L B$, respectively. The duality gap between $U B$ and $L B$, computed by $|(U B-L B) / L B| * 100 \%$, illustrates the optimality of problem solution. The smaller duality gap computed, the better the optimality.

A LR-based primal heuristic algorithm is listed in Figure 5 and object tracking tree algorithm is listed in Figure 6. 


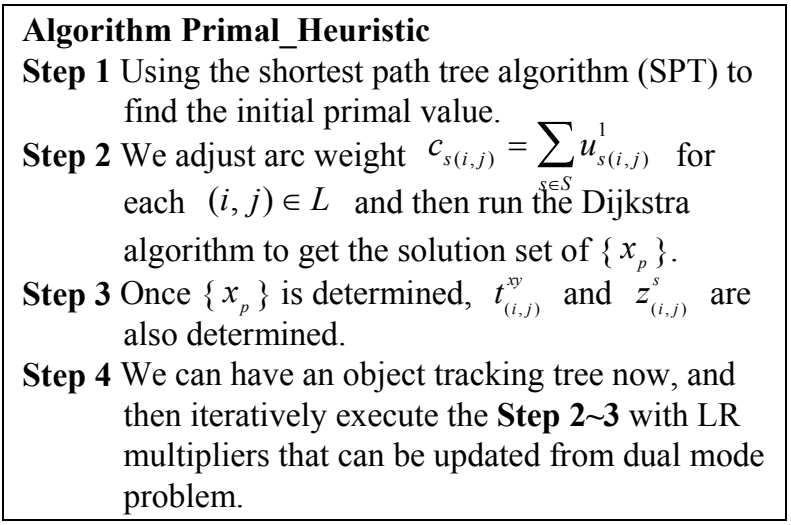

Fig. 5: The LR-based primal heuristic algorithm.

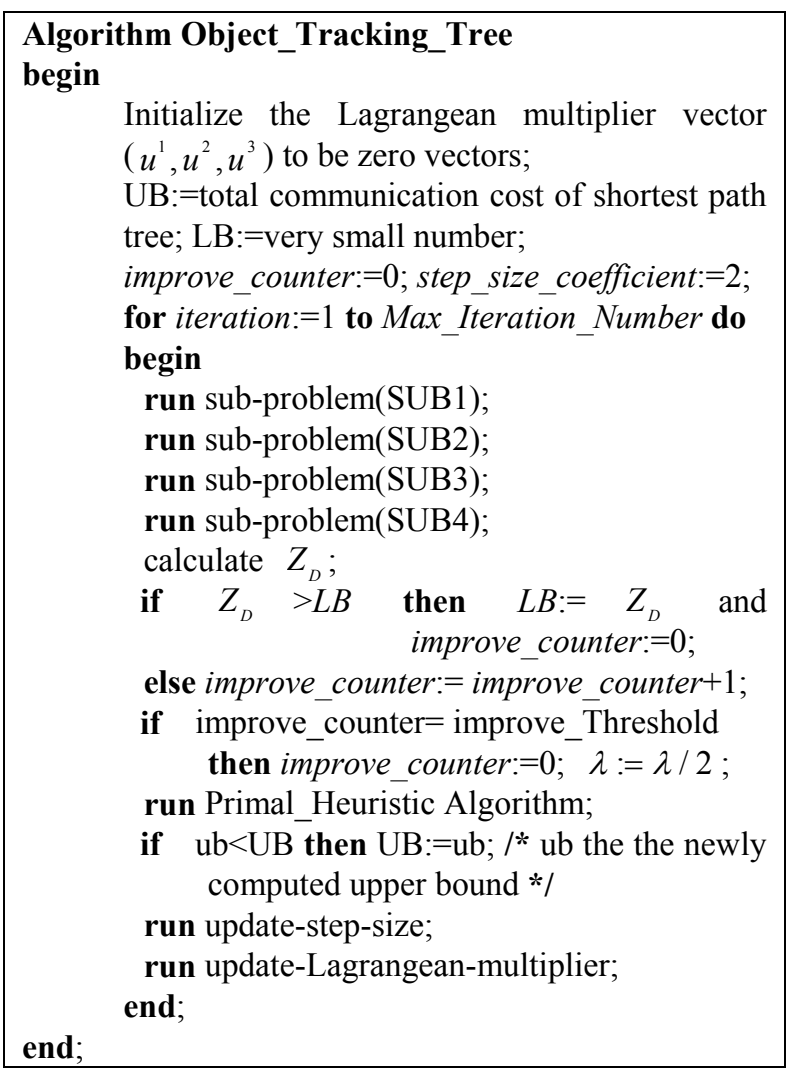

Fig. 6: The object tracking tree algorithm.

\section{Computational Experiments}

To evaluate the performance of the proposed algorithm, we conduct an experiment. The performance is assessed in terms of total communication cost.

\subsection{Scenario}

The proposed algorithm is coded in $\mathrm{C}$ under a Microsoft Visual $\mathrm{C}++6.0$ development environment.
All the experiments are performed on a Pentium IV-2.8G Hz PC running Microsoft Windows XP. The algorithm is tested on a $2 \mathrm{D}$ sensor field. We distribute $|S|=12$ sensor nodes and $|C|=11$ communication nodes in sensor field, as shown in Figure 2 and 3.

\subsection{Experimental Results}

In order to evaluate our proposed heuristic algorithm, we compare this one with other heuristic algorithm, such as shortest path tree (SPT) algorithm. With the exception of this SPT algorithm, the proposed heuristic algorithm can be also compared with dual mode problem value (Low Bound, $L B$ ).

Figure 7 shows an example of LR-based object tracking tree.

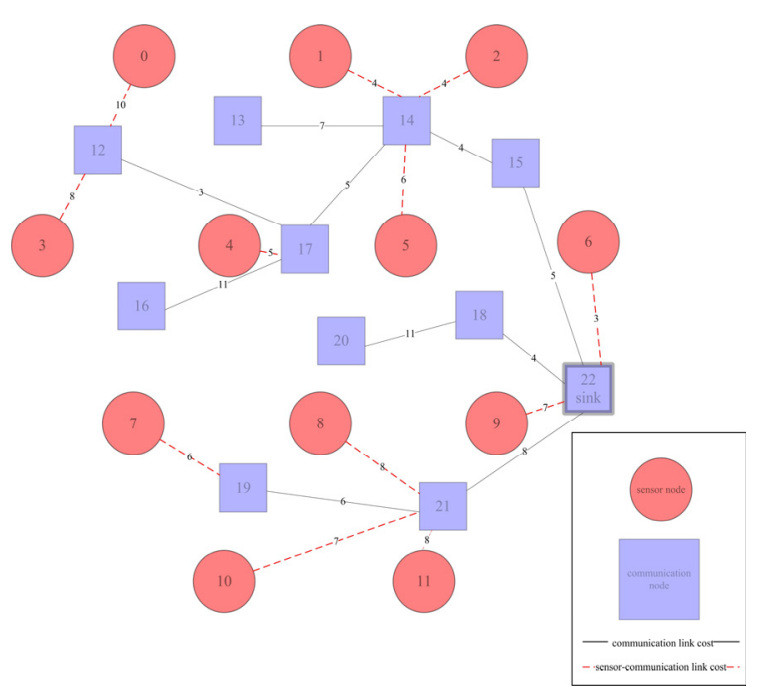

Fig. 7: Example of LR-based object tracking tree.

Figure 8 shows an example of the trend line for getting the primal problem solution values $(U B)$ and dual mode problem value $(L B)$. The $U B$ curve tends to decrease to get the minimum feasible solution. In contrast, the $L B$ cure tends to increase and converge rapidly to reach the optimal solution. The LR-based method ensures the optimization results between the $U B$ and $L B$ so that we can keep the duality gap as small as possible in order to improve our solution quality and achieve near optimization. In this example, the duality gap between $U B$ and $L B$ is $1.2 \%$.

This study shows the best found for the minimum total communication cost by the proposed algorithm. Eventually, the total communication cost of SPT algorithm is 5019, the total communication cost of LR-based algorithm $(U B)$ is 4568 , and the dual mode problem value $(L B)$ is 4513 . 


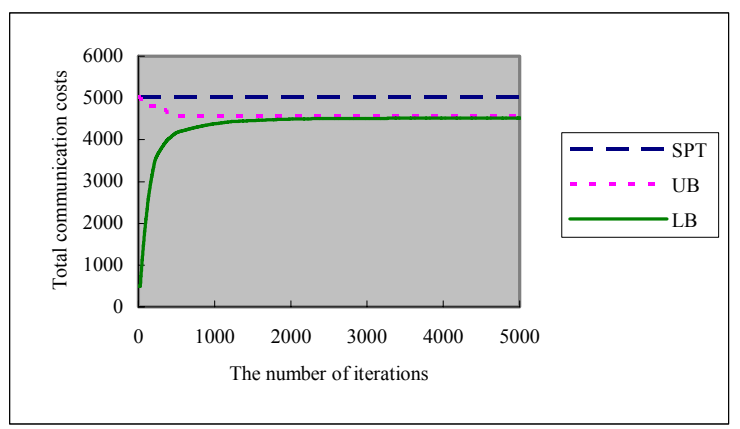

Fig. 8: The execution results of LR-based algorithm.

Table 1 shows that the time complexity of our LR-based solution is dominated by Lagrangean dual problem. The Lagrangean dual problem is to solve the above four sub-problems with the maximum number of iteration $I$.

Table 1: The time complexity of our proposed LR-based object tracking tree algorithm

\begin{tabular}{|cc|}
\hline \multicolumn{2}{|c|}{ LR-based solution approach } \\
\hline Sub-problem & Time complexity \\
\hline Sub-problem (SUB1) & $O\left(|S|^{2}|L|\right)$ \\
Sub-problem (SUB2) & $O(|S||L|)$ \\
Sub-problem (SUB3) & $O\left(|S|^{2}|L|\right)$ \\
Sub-problem (SUB4) & $O\left(|S|^{2}|L|\right)$ \\
Lagrangean dual problem & $O\left(I|S|^{2}|L|\right)$ \\
\hline *Where parameter I mean the maximum number of iterations \\
\hline
\end{tabular}

\section{Conclusions}

This study proposes an object tracking algorithm in wireless sensor networks. To our best knowledge, the proposed algorithm is truly novel and it has not been yet discussed in previous researches. This study first formulates the problem as a $0 / 1$ integer programming problem, and then proposes a Lagrangean relaxation-based heuristic algorithm for solving the optimization problem.

The experimental results show that the algorithm is not only better than the other heuristic algorithm, such as SPT, but the duality gap is also small. That is, the proposed heuristic algorithm can improve about $9.9 \%$ energy consumption when compared with SPT and achieve the near optimal solution since the duality gap is only $1.2 \%$. Therefore, the results show that the proposed LR-based algorithm can achieve efficient object tracking. Furthermore, the algorithm is very efficient and scalable in terms of the solution time.

As to the next step, we plan to further investigate response time model based on object tracking application requirements and heuristic algorithms. In addition, we are looking into the tradeoff of total communication cost with various system issues, such as response time, report frequency, number of sinks, etc.

\section{References}

[1] H.T. Kung and D. Vlah, "Efficient Location Tracking Using Sensor Networks," in Proc. IEEE Wireless Communications and Networking Conference (WCNC), 2003.

[2] Chih-Yu Lin and Yu-Chee Tseng, "Structures for In-network Moving Object Tracking in Wireless Sensor Networks," in Proc. IEEE First International Conference on Broadband Networks (BROADNETS), 2004.

[3] I. F. Akyildiz, W. Su, Y. Sankarasubramaniam, and E. Cayirci, "Wireless Sensor Networks: a Survey," Elsevier Journal of Computer Networks, vol. 38, pp. 393-422, March 2002.

[4] I. F. Akyildiz, W. Su, Y. Sankarasubramaniam, and E. Cayirci, "A Survey on Sensor Networks," IEEE Communications Magazine, pp. 102-114, August 2002.

[5] M. L. Fisher, "The Lagrangean Relaxation Method for Solving Integer Programming Problem," Management Science, vol. 27, no. 1, pp. 1-18, Jan. 1981.

[6] M. L. Fisher, "An Applications Oriented Guide to Lagrangian Relaxation," Interfaces, 15:2, pp. 10-21, March-April 1985.

[7] Yingqi Xu; Wang-Chien Lee, “On Localized Prediction for Power Efficient Object Tracking in Sensor Networks," Proceedings of the $23 \mathrm{rd}$ International Conference on Distributed Computing Systems Workshops (ICDCSW), 2003).

[8] Yingqi Xu; Winter, J.; Wang-Chien Lee, "Prediction-based strategies for energy saving in object tracking sensor networks," Mobile Data Management, 2004. Proceedings. 2004 IEEE International Conference on Mobile Data Management (MDM, 2004), 2004, pp. 346 357.

[9] Yingqi Xu; Winter, J.; Wang-Chien Lee, "Dual prediction-based reporting for object tracking sensor networks, " The First Annual International Conference on Mobile and Ubiquitous Systems: Networking and Services (MobiQuitous), 2004), Aug. 22-26, 2004, pp. 154 - 163. 